\title{
Study on the Diagnosis of Hysteroscopic Uterine Bleeding
}

\author{
Xiaojun Liu ${ }^{1}$, Jiawen Zhou*1 \\ ${ }^{1}$ China-Japan Union hospital of Jilin University, Changchun, Jilin, 130033
}

Keywords: bleeding diagnosis; Hysteroscopic Uterine; medicine effect

\begin{abstract}
To analyze the clinical application effect of hysteroscopy in diagnosis and treatment of abnormal uterine bleeding, a total of 112 patients with abnormal uterine bleeding diagnosed in our hospital from November 2014 to November 2015 were selected, aged from 19 to 58 years, with an average age of (30.3 \pm 4.32$)$ years. The disease duration ranged from 2 months to 6 years; Bleeding types include abnormal menstrual bleeding, prolonged menstruation, abnormal menstrual bleeding, and abnormal vaginal bleeding after menopause. Hysteroscopic techniques were used to treat these patients. After treatment, the clinical effects of hysteroscopy in abnormal uterine bleeding were analyzed and compared. A total of 112 patients with abnormal uterine bleeding were found in the study and 70 patients were diagnosed as positive by ultrasound. The positive rate was $62.5 \%$. The positive rate of hysteroscopy was 83 . The positive rate was $74.1 \%$. After electrotransplantation of 43 patients with endocervical polyps using uterine lesions, 30 patients were cured, and the rate of improvement in symptoms was $69.77 \%$; 30 patients with cervical polyps were cured in 23 cases. The proportion of symptom improvement was 76.67\%; 22 cases of uterine fibroids were all cured, and the improvement of symptoms reached 100\%. Therefore, hysteroscopy has a significant clinical effect in the diagnosis and treatment of abnormal uterine bleeding, can accurately diagnose the cause of the disease and has achieved certain results in the treatment with value in clinical use and promotion.
\end{abstract}

\section{Introduction}

Abnormal uterine bleeding is a common gynecological disease of modern women. This disease often occurs in miscarriage or intrauterine diseases. At present, hysteroscopic techniques are mostly used to treat abnormal uterine bleeding. Hysteroscopy is a minimally invasive procedure. The main purpose of this study was to analyze the clinical effect of hysteroscopy in the diagnosis and treatment of abnormal uterine bleeding. A total of 112 patients with abnormal uterine bleeding who were present in our hospital from November 2014 to November 2015 were selected as this The subjects of the study were those who received very satisfactory results after undergoing hysteroscopic treatment. The specific reports are as follows.

\section{Materials and Methods}

Normal information. A total of 112 patients with abnormal uterine bleeding diagnosed in our hospital from November 2014 to November 2015 were selected, aged from 19 to 58 years, with an average age of $(30.3 \pm 4.32)$ years; duration of disease from 2 months to 6 years; bleeding in these patients. Types include irregular menopausal bleeding, prolonged menstruation, abnormal menstrual bleeding, and abnormal vaginal bleeding after menopause.

Standard constrain. The clinical manifestations of these patients were: 1 non-normal menstrual bleeding caused by external vaginal and cervical factors; 2 no use of hormone supplementation within 2 years; 3 no threatened abortion; 4 exclusion of acute pelvic inflammatory disease.

Treatment method. All 112 patients were treated with hysteroscopy. The specific methods were as follows: (1) Prepare preoperatively. After the patient was admitted to the hospital, the relevant nursing staff should do the preoperative cleaning of the patient. Vaginal cleansing should be strictly prohibited before eating. (2) The specific surgical treatment process requires the patient to perform vaginal color ultrasound examination before surgery, perform hysteroscopy before surgery, and 
sterilize the patient's external vaginal cavity. Necessary anesthesia of the cervical canal is performed with a cervical tube iodophor swab. Mirror examination, if the cervix is normal, perform a curettage immediately. If a hysteroscopy reveals that there is a polyp in the uterus, submucous myoma of the uterus, or a polyp in the cervix, an intravenous anesthesia is required for the patient to perform an electrotomy of the polyp in the endocervical canal. If the preoperative ultrasound shows that the uterine cavity or near the uterine cavity has obvious space-occupying lesions, when the patient is treated, epidural anesthesia should be selected and the patient should be given hysteroscopy according to the specific examination results. Uterine lesion resection.

Statistical method. All data were statistically analyzed. Percentages (\%) represent count data, and $\chi 2$ were tested. Statistical results were statistically different at $\mathrm{P}<0.05$.

\section{Results and Discussion}

Comparison of the positive rate of ultrasound and hysteroscopy. Of the 112 patients with abnormal uterine bleeding, 70 were positive for ultrasound examination, the positive rate was $62.5 \%$, and 83 were positive for hysteroscopy. The positive rate was $74.1 \%(\mathrm{P}<0.05)$, which was statistically significant.

Abnormal uterine bleeding is a common disease in gynecology. The patient will experience abnormal menstrual bleeding, which will have a great impact on the patient's life and reduce the quality of life of the patient. At present, the use of hysteroscopic treatment has improved the therapeutic effect and it has been able to detect the small intrauterine lesions that cannot be detected by B ultrasound [1]. In this study, hysteroscopic treatment methods were introduced. Before the operation of the patient, the patient could be examined and diagnosed by hysteroscopy, and the lesions of the intrauterine and cervical canal were clearly inspected and the patient was promptly treated. The use of hysteroscopic techniques for treatment is more accurate and reliable in the examination and treatment than traditional treatment of abnormal uterine bleeding. This not only improves the accuracy of diagnosis, but also greatly improves the efficacy of treatment. More comprehensive services for patients [2]. Hysteroscopic treatment of abnormal uterine bleeding using minimally invasive surgical techniques, compared with the traditional B-ultrasound, its greatest advantage is the ability to observe and treat the uterine cavity directly, to identify the cause of the disease at one time, to take the cause The appropriate treatment. In the present study, 112 patients with abnormal uterine bleeding were treated with hysteroscopy. In the study investigation, 112 patients with abnormal uterine bleeding and 70 patients with positive ultrasound examination were found. The positive rate was $62.5 \%$. The positive rate of microscopic examination was 83 cases, and the positive rate was $74.1 \%$. After electrotransplantation of 43 patients with endocervical polyps using uterine lesions, 30 patients were cured. The rate of improvement in symptoms was 69.77\%; 30 cases of cervical polyps were cured in 23 cases, and the rate of symptom improvement was $76.67 \%$. All 22 cases of uterine fibroids were cured, and the improvement of symptoms reached $100 \%$.

\section{Clinical Diagnosis and Pathological Comparison}

The total coincidence rate of diagnosis and pathological results in the hysteroscopy group and the curettage group was $89.6 \%$ and $67.9 \%$, which was significantly different from that of heterogeneous sciences, $\chi 2=12.76, \mathrm{P}<0.05$ (Table 1$) .10$ cases $(10.4 \%)$ of pathological findings were inconsistent with hysteroscopic diagnosis: 3 cases of endometrial hysteroscopy were diagnosed as having 3 cases of intimal hyperplasia, 1 case of endometrial polyps, and 3 cases of atrophy. One case of endometrial hyperplasia was diagnosed hysteroscopically, and 2 cases were proliferative endometrium; 1 case of endometrial simple hyperplasia was diagnosed as endometrial polyp under hysteroscopy; 1 case was diagnosed with uterine misdiagnosis of endometrial dysplasia. Endometrial cancer; 1 case of endometrial polyps misdiagnosed as uterine fibroids; 1 case of endometrial cancer misdiagnosed as endometrial polyps. Among the 62 patients with successful curettage, 7 cases of scratched pathology were inconsistent with the final pathology: 2 cases with 
atrophic intima were diagnosed with endometrial polyps by hysteroscopic resection and 1 case was diagnosed as endometritis with hysteroscopy. A biopsy showed a simple hyperplasia of the intima; 1 patient had a hysteroscopic biopsy of the endometrial polyps as a dysplasia of the intima (after surgery was confirmed); 1 patient had a hysteroscopic biopsy of the submucosal myoma Cancer (after surgery confirmed); Endometrial dysplasia in 1 case diagnosed with endometrial cancer by hysteroscopy and hospitalization; 2 cases of complex hyperplasia confirmed by hysteroscopy and hospitalization in 1 case of endometrial cancer, 1 case Still a proliferation of complexity.

Diagnosis and curettage is a traditional diagnostic method for PMB. Because it is a blind curettage, it is easy to miss small lesions behind the uterine horn or uterine space occupying lesions such as submucosal myoma. The hysteroscope can directly look into the uterine cavity structure and enlarge with the help of the lens. The effect identifies focal endometrial lesions, and can also perform microscopic biopsy of suspicious lesions. Angioni et al. [4] studied the results of 390 cases of $\mathrm{PMB}$, showing that the compliance rate of the curettage and pathological diagnosis was slightly consistent, while hysteroscopy was almost identical. The total coincidence rate of hysteroscopy and pathology in this study was much higher than that of diagnostic curettage group. Intrauterine space-occupying lesions such as endometrial polyps and submucosal myoma are difficult to accurately determine. The small polyps at the corners of palaces and palaces are easy to leak, and the thick polyps at the pedicles are not easy to scrape. Uterine fibroids can only be inferred from the irritability of the uterine cavity during curettage. Hysteroscopy can visually see the size, shape and growth location of the two. The coincidence rate between microscopic and postoperative pathology is higher than that of diagnostic curettage group. Atrophic endometrium and endometritis are not easy to obtain pathology due to thinning of the endometrium, and full scuffing can also cause inflammation of the intima. The hysteroscopic characteristics of the two are more obvious: atrophic endometrial uterine cavity morphology is smaller, the inner film is thin and smooth, white and yellow; endometritis, the endometrial membrane is red, with patches of congestion spots, showing red and white. The diagnostic accuracy and pathology of the two were $90.3 \%$ and $100 \%$. Visible to endometrial contraction and endometriosis, hysteroscopy can be diagnosed, if necessary, can be combined with local biopsy confirmed by microsurgery, avoid unnecessary curettage. Endometrial cancer and endometrial hyperplasia, especially the early endometrial cancer lesions are more limited and located at the bottom of the uterus and at the uterine horn. Blind diagnosis and cure can easily cause missed diagnosis. The hysteroscopy can be seen directly in some of the endometrial cancer tissue was gray or grayish yellow, hyperplasia or prominent, some diffuse involvement of the entire uterine cavity with vasodilation disorders, tissue brittle surface Contamination, sometimes visible spotted bleeding and necrosis. There were 8 cases of endometrial cancer in the diagnostic curettage group, of which 5 cases were diagnosed with curettage. The other 3 cases (intimal dysplasia, complex hyperplasia, and 1 submucosal myoma) were all diagnosed as misdiagnosed after surgery. In the hysteroscopic group, only one endometrial cancer was misdiagnosed as endometrial polyps. It can be seen that hysteroscopy can directly observe the location and extent of cancer foci, which is beneficial to the preoperative staging and the choice of surgical methods and scope [5]. Hysteroscopy is a morphological examination, and although the tissue is enlarged many times, it still cannot replace the pathological diagnosis. The results of this study show that hysteroscopy has a good recognition of morphological features such as submucosal fibroids and polyps, high diagnostic accuracy, and proliferative endometrium, endometrial folds, intimal hyperplasia Symptoms and early endometrial cancer due to morphological characteristics are not obvious under microscope is sometimes difficult to identify. In this study, 1 case of hysteroscopic endometrium showed polypoid protrusions, gray and white crisp, endoscopic cancer diagnosis of endometrial cancer, microscopic biopsy pathology showed endometrial dysplasia, hospitalized surgical excision of the uterus Pathology is still atypical hyperplasia, confirmed as a misdiagnosis under the microscope. One patient underwent endoscopic biopsy with hysteroscopic polypectomy for endometrial biopsy. The pathological findings showed endometrial cancer. The uterus was removed surgically. The pathology was focal endometrioid adenocarcinoma. Visible hysteroscopy must be combined with biopsy to make the diagnosis more complete. 


\section{Conclusion}

Hysteroscopic minimally invasive techniques have significant clinical effects in the diagnosis and treatment of abnormal uterine bleeding and are worthy of promotion.

\section{References}

[1] Wang Ruige. Clinical value of hysteroscopy in diagnosis and treatment of abnormal uterine bleeding [J]. Chinese Journal of Clinical Research, 2011, 07 (24): 598-599.

[2] Wang Ying, Xu Cailin, Cao Yanhua, Chen Ying. Hysteroscopic technique in the diagnosis and treatment of patients with abnormal uterine bleeding [J]. Journal of Hainan Medical College, 2015, 04 (21): 527-529+533.

[3] Yang Qing, Zhang Ming. The clinical significance of hysteroscopy in the diagnosis of abnormal uterine bleeding [J]. Liaoning Medical Journal, 1999 (3): 145-146.

[4] Zhang Ke-qun. Ultrasound diagnosis of curettage and hysteroscopy in the diagnosis of postmenopausal bleeding [J]. Chinese Journal of Practical Diagnosis and Therapy, 2011, 25 (5): 488-489.

[5] Shi Hongqi, Hu Yi, Zhou Yibo. The use of hysteroscopy and segmented curettage in the diagnosis of postmenopausal vaginal bleeding [J]. Clinical Medicine, 2004, 24(6):54-56. 